\title{
The Kirkwood-Salsburg Equations: Solutions and Spectral Properties
}

\author{
Herm Jan Brascamp ${ }^{\star}$ \\ The Institute for Advanced Study, Princeton, New Jersey, USA
}

Received July 7, 1974

\begin{abstract}
It is shown that the Kirkwood-Salsburg equations for a classical lattice gas are equivalent to the Dobrushin-Lanford-Ruelle equilibrium equations. The term "Kirkwood-Salsburg equations" is used here in a restricted sense, and thus the known result for a larger system of equations is improved (see Table 1). Some information on the spectrum of the Kirkwood-Salsburg operator is found in connection with zeros of partition functions. An example is given to show that the Kirkwood-Salsburg equations can have other solutions than states in the space of uniformly bounded correlation functions.
\end{abstract}

\section{Introduction and Notation}

Let us first briefly recall some by now standard notation concerning interactions and states for a classical lattice gas (see e.g. [1,2]). In this paper, we mean by a lattice $L$ nothing more than a countable (finite or infinite) number of points. A configuration is a subset $X \subset L$, where the points in $X$ are called occupied, the points outside $X$ empty.

For two sets $X, Y$ with $Y \subset X$, we shall denote the difference by $X-Y$. Further we shall not distinguish in notation between a lattice point $x$ and the set consisting of that point only.

An interaction is a map $\Phi$ from the finite, non-empty sets $Y \subset L$ to the real numbers with the property

$$
\sum_{Y: x \in Y}|\Phi(Y)|<\infty, \quad \forall x \in L .
$$

The energy of a finite set $X$, given a fixed configuration $S \subset L-X$, is then

$$
W(X \mid S)=\beta \sum_{\substack{Y \subset X \cup S \\ Y \cap X \neq \emptyset}} \Phi(Y)-\beta \mu|X|,
$$

where $|X|$ is the number of points in $X$. For $S=\emptyset$, one usually writes $W(X \mid \emptyset)$ $=U(X)$. The inverse temperature $\beta$ and the chemical potential $\mu$ are absorbed in $W$ for notational convenience.

By Eq. (1), $|W(X \mid S)|<\infty$. Moreover, with $S_{M}=S \cap M$,

$$
\lim _{M \rightarrow L-X} W\left(X \mid S_{M}\right)=W(X \mid S)
$$

\footnotetext{
* Supported by National Science Foundation Grant GP-40768X.
} 
uniformly in $S$. (In precise terms, the notation in Eq. (3) means the following: For all $\varepsilon>0$ there exists a finite set $K \subset L-X, K$ independent of $S$, such that

$$
\left|W\left(X \mid S_{M}\right)-W(X \mid S)\right|<\varepsilon
$$

for all finite $M \subset L-X$ with $M \supset K$.)

In particular, let the interaction be of finite range, which means that the set $M(X)$ of points, interacting with a finite set $X$, is finite. Then, for all $M \supset M(X)$,

$$
W(X \mid S)=W\left(X \mid S_{M(X)}\right)=W\left(X \mid S_{M}\right) .
$$

Note further, that for finite $S$

$$
W(X \mid S)=U(X \cup S)-U(S) .
$$

Linear relations between quantities $W$ can conveniently be checked with the help of this relation, even if $S$ is infinite.

A state on $L$ can be characterized in a number of ways:

By the generalized correlation functions $\left\{\mu_{\Lambda}(X), \Lambda\right.$ finite, $\left.X \subset \Lambda\right\}, \mu_{\Lambda}(X)$ being the probability that the points in $X$ are occupied and that the points in $\Lambda-X$ are empty. The $\mu_{\Lambda}(X)$ must be non-negative and satisfy the consistency relations, for $\Lambda \subset M$,

$$
\sum_{Y \subset M-\Lambda} \mu_{M}(X \cup Y)=\mu_{\Lambda}(X)
$$

By the correlation functions $\varrho(X)=\mu_{X}(X)$, with the converse relation

$$
\mu_{\Lambda}(X)=\sum_{Y \subset A-X}(-1)^{|Y|} \varrho(X \cup Y) .
$$

As a positive linear form on the algebra $C$ of continuous functions of configurations.

An equilibrium state corresponding to the interaction $\Phi$ is defined as a state satisfying the DLR (Dobrushin [3]-Lanford-Ruelle [4]) equilibrium equations, which can be expressed as follows:

There is some increasing sequence of finite sets $\{\Lambda\}$, with $\Lambda \rightarrow L$, such that the equations

$$
\begin{aligned}
\lim _{M \rightarrow L-\Lambda} \mu_{\Lambda \cup M}\left(X \cup S_{M}\right) / \mu_{M}\left(S_{M}\right) & =\exp [-W(X \mid S)] / Z_{\Lambda}(S), \\
Z_{\Lambda}(S) & =\sum_{Y \subset \Lambda} \exp [-W(Y \mid S)]
\end{aligned}
$$

hold for all $\Lambda, X \subset \Lambda, S \subset L-\Lambda$.

For finite range interactions, the equations are simplified to [cf. Eq. (4)]

$$
\mu_{\Lambda \cup M(\Lambda)}(X \cup S) / \mu_{M(\Lambda)}(S)=\exp [-W(X \mid S)] / Z_{\Lambda}(S)
$$

Let us now give various systems of equations for the correlation functions, which are closely related to the DLR equations. For the lattice gas, correlation equations were first given by Gallavotti and Miracle-Sole [5]. 
By the Mayer equations we designate the following family of equations:

where

$$
\begin{aligned}
\varrho(\emptyset) & =1 ; \\
\varrho(X) & =\sum_{T \subset L-X} \sum_{S \subset X-Y}(-1)^{|S|} \varrho(Y \cup S \cup T) K(X, Y, T),
\end{aligned}
$$

$$
K(X, Y, T)=\sum_{R \subset T}(-1)^{|T-R|} \exp [-W(X-Y \mid Y \cup R)],
$$

and where $X, Y$ are finite sets with $Y \subset X, Y \neq X$. In Eq. (10), $T$ runs through the finite subsets of $L-X$.

A number of subfamilies of (10) is introduced by putting further restrictions on the pair $(X, Y)$, see Table 1 .

Table 1

\begin{tabular}{lll}
\hline Symbol & Name & Restriction \\
\hline M & Mayer & - \\
EKS & Extended Kirkwood-Salsburg & $|Y|=|X|-1$ \\
KS & Kirkwood-Salsburg & $Y=X-x, x$ first point in $X$ \\
MM & Mayer-Montroll & $Y=\emptyset$ \\
\hline
\end{tabular}

To obtain the KS equations, an order has to be introduced on $L ; Y$ is then obtained from $X$ by removing the first point.

We trivially have the implications of the following scheme.

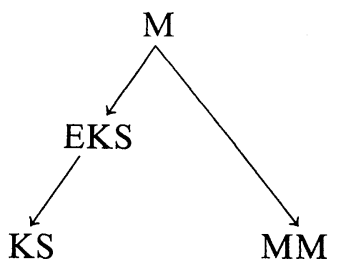

In fact, EKS is equivalent to $M$ ([2]; the analogous statement for continuous systems has been proved in [6], for polymer systems in [7]). Also, in [2, 8] (and $[6,8]$ for continuous systems) the EKS equations were shown to be equivalent to the DLR equations.

Still, the family EKS seems superfluous; it is tempting to consider instead families, which have (for finite $L$ ) as many equations as unknowns. This requirement is satisfied by the $\mathrm{KS}$ and MM equations. For the lattice gas, the MM equations are quite unsatisfactory:

Proposition 1. The MM equations may have other states than equilibrium states as a solution.

Proof. Let $L$ consist of two points, and let $\Phi=0, \mu=0$. Then the MM equations are

$$
\begin{aligned}
\varrho(1) & =1-\varrho(1) \\
\varrho(2) & =1-\varrho(2) \\
\varrho(12) & =1-\varrho(1)-\varrho(2)+\varrho(12) .
\end{aligned}
$$


Thus, $\varrho(1)=\varrho(2)=\frac{1}{2}$, but $\varrho(12)$ is undetermined. For the equilibrium state, $\varrho(12)=\frac{1}{4}$.

Q.E.D.

The KS equations are better behaved: in Section 2 we show that all states satisfying the KS equations are equilibrium states. It should be noted, that the proof strongly depends on the fact, that $L$ has a first element in its order. Therefore the question remains open if the order is such that $L$ extends to $\infty$ at both ends.

Let us write out the KS equations: $\varrho(\emptyset)=1$;

$\varrho(X)=\sum_{T \subset L-X}\left\{\varrho\left(X_{1} \cup T\right)-\varrho(X \cup T)\right\} \sum_{R \subset T}(-1)^{|T-R|} \exp \left[-W\left(x \mid X_{1} \cup R\right)\right]$,

where $X_{1}$ is obtained by removing the first point, $x$, from $X$. Let us note in passing that the right member of Eq. (12) can be conveniently rewritten as

$$
\varrho(X)=\lim _{M \rightarrow L-X} \sum_{Y \subset M} \mu_{X \cup M}\left(X_{1} \cup Y\right) \exp \left[-W\left(x \mid X_{1} \cup Y\right)\right] .
$$

In Section 3, arbitrary complex values for the fugacity $z=\exp (\beta \mu)$ are considered. A connection between the zeros of the partition function, the spectrum of the KS operator and non-uniqueness of the equilibrium state is found. To elucidate this point, note that Eq. (12) can be written in a vector form

$$
\varrho=\alpha+z \boldsymbol{K} \varrho,
$$

where the KS operator $\boldsymbol{K}$ does not depend on $z$. As usual, we shall consider Eq. (14) on the Banach space $B$ of vectors $\{\varrho(X)\}$ with norm

$$
\sup _{X}|\varrho(X)|<\infty .
$$

Obviously, if the equilibrium state is not unique for some value of $z$, Eq. (14) has more than one solution, and the point spectrum of $\boldsymbol{K}$ contains the point $z^{-1}$. As Theorem 4 (ii) shows, $z^{-1}$ is in the spectrum of $\boldsymbol{K}$, if $z$ is a limit of zeros of partition functions.

A serious drawback against the space $B$ is the following: For positive, real $z$ it may happen, that the equilibrium state is unique, whereas the KS equations have more solutions in $B$ (not representing states); then again, $z^{-1}$ is in the point spectrum of $\boldsymbol{K}$ (see Section 4, Example 1).

Therefore it may seem natural to consider instead of $B$ a smaller space, namely the space of bounded linear functionals, $C^{*}$, on the algebra $C$ of continuous functions of configurations. The corresponding norm is

$$
\sup _{\Lambda} \sum_{X \subset A}\left|\mu_{\Lambda}(X)\right| .
$$

However, the space $C^{*}$ is completely unsatisfactory for the consideration of the spectrum of $\boldsymbol{K}$ : On $C^{*}, \boldsymbol{K}$ is not bounded, and if $z$ is not positive real, Eq. (14) has generally no solution at all in $C^{*}$. Then the spectrum of $\boldsymbol{K}$ consists of the whole complex plane. The intuitive reason for that is, that for an "equilibrium functional"

$$
\sum_{X \subset \Lambda}\left|\mu_{\Lambda}(X)\right| \approx Z_{\Lambda}(|z|) /\left|Z_{\Lambda}(z)\right|,
$$


which grows exponentially with $|\Lambda|$. For example, if $\Phi=0$,

$$
\sum_{X \subset A}\left|\mu_{\Lambda}(X)\right|=\{(1+|z|) /|1+z|\}^{|\Lambda|} .
$$

Thus $B$ seems to be the only reasonable space in which to study the spectrum of $\boldsymbol{K}$.

One could try to circumvent the study of the spectrum of $\boldsymbol{K}$ and establish a connection between the zeros of the partition function and non-uniqueness of the equilibrium state, as the results in Section 3 suggest. A further negative result is however, that non-uniqueness of the equilibrium state for a positive, real $z$ neither implies, nor is implied by, the fact that $z$ is a limit of zeros of partition functions (see Examples 2 and 3, Section 4).

\section{The Equivalence of the KS Equations and the DLR Equations}

In this section we restrict ourselves to positive, real $z$ and to states. On the lattice $L$ an order is introduced, so that $L=\{1,2,3, \ldots\}$. Henceforth, we shall write $Z_{k}(S)$ for $Z_{[1, k]}(S)$, Eq. (8).

Theorem 2. For a lattice gas with arbitrary many body potentials, only restricted by Eq. (1), all states satisfying the $\mathrm{KS}$ equations are equilibrium states.

Proof. The proof will be given in three steps: First for finite $L$, next for infinite $L$ with finite range interactions, and finally for arbitrary interactions.

Step 1: consists of the following lemma.

Lemma 3. For finite $L=\{1,2,3, \ldots, n\}$ and any interaction, the KS equations have the Gibbs state,

as the only solution.

$$
\mu_{L}(X)=\exp [-U(X)] / Z_{n},
$$

Proof. Instead of the $2^{n}$ unknowns $\varrho(X), X \subset L$, it is more convenient to use the $\mu(X) \equiv \mu_{L}(X)$. In terms of these unknowns, the KS equations read [cf. Eq. (13)]

$$
\begin{aligned}
\sum_{Y \subset L} \mu(Y) & =1 ; \\
\sum_{Y \subset L-X} \mu(X \cup Y) & =\sum_{Y \subset L-X} \mu\left(X_{1} \cup Y\right) \exp \left[-W\left(x \mid X_{1} \cup Y\right)\right], \quad X \neq \emptyset .
\end{aligned}
$$

Let us regroup the $2^{n}-1$ Eq. (17). If $X \neq \emptyset, X \subset[1, n-1]$, then $(X \cup n)_{1}=X_{1} \cup n$. Therefore the equation for $X \subset[1, n-1]$ contains all terms occurring in the equation for $X \cup n$, so that the latter can conveniently be subtracted from the former. We get

$$
\begin{aligned}
& \sum_{Y \subset[1, n-1]-X} \mu(X \cup n \cup Y)= \sum_{Y \subset[1, n-1]-X} \mu\left(X_{1} \cup n \cup Y\right) \exp \left[-W\left(x \mid X_{1} \cup n \cup Y\right)\right], \\
& X \subset[1, n-1], \quad X \neq \emptyset ; \\
& \sum_{Y \subset[1, n-1]-X} \mu(X \cup Y)=\sum_{Y \subset[1, n-1]-X} \mu\left(X_{1} \cup Y\right) \exp \left[-W\left(x \mid X_{1} \cup Y\right)\right], \\
& X \subset[1, n-1], \quad X \neq \emptyset ; \\
& \sum_{Y \subset[1, n-1]} \mu(Y \cup n)=\sum_{Y \subset[1, n-1]} \mu(Y) \exp [-W(n \mid Y)] .
\end{aligned}
$$


Note, that Eqs. (18) and (19) have the form of Eq. (17), but with $[1, n]$ replaced by $[1, n-1]$ and energies $W(X \mid n)$ and $U(X), X \subset[1, n-1]$, respectively. Thus, if we assume Lemma 3 to be true for $n-1$ points, the $2^{n}-1$ Eqs. (16) and (18)-(19) imply

$$
\begin{aligned}
\mu(X \cup n) & =\varrho(n) \exp [-W(X \mid n)] / Z_{n-1}(n), X \subset[1, n-1] ; \\
\mu(X) & =[1-\varrho(n)] \exp [-U(X)] / Z_{n-1}, X \subset[1, n-1] .
\end{aligned}
$$

The remaining unknown now follows by substituting these expressions into Eq. (20):

or

$$
\varrho(n)=[1-\varrho(n)] \exp [-U(n)] Z_{n-1}(n) / Z_{n-1},
$$

$$
\varrho(n)=\exp [-U(n)] Z_{n-1}(n) / Z_{n} \text {. }
$$

This proves Lemma 3 for $n$ points, assuming that it holds for $n-1$ poinzs. Since the lemma is obvious for $n=1$, the proof is concluded by induction.

Step 2: We now consider the KS equations on an infinite lattice $L=\{1,2,3, \ldots\}$ with finite range interactions.

Fix a natural number $n$, and take $\Lambda=[1, n]$. Let further $M$ consist of all points outside $\Lambda$, interacting with $\Lambda$; by definition, $M$ is finite.

If $X \subset A, X \neq \emptyset$, and $S \subset M$, then $(X \cup S)_{1}=X_{1} \cup S$. Therefore the KS equation starting with $\varrho(X \cup S)$ reads [cf. Eqs. (13) and (4)]

$$
\begin{aligned}
& \sum_{\substack{Y \subset A-X \\
T \subset M-S}} \mu_{\Lambda \cup M}(X \cup Y \cup S \cup T) \\
& \quad=\sum_{\substack{Y \subset A-X \\
T \subset M-S}} \mu_{\Lambda \cup M}\left(X_{1} \cup Y \cup S \cup T\right) \exp \left[-W\left(x \mid X_{1} \cup Y \cup S \cup T\right)\right],
\end{aligned}
$$

wher again it is more convenient to use quantities $\mu$ instead of $\varrho$. The equations for fixed $X$ and different sets $S$ can be combined, as in Step 1, to give

$$
\begin{aligned}
\sum_{Y \subset A-X} \mu_{\Lambda \cup M}(X & \cup Y \cup S) \\
& =\sum_{Y \subset A-X} \mu_{\Lambda \cup M}\left(X_{1} \cup Y \cup S\right) \exp \left[-W\left(x \mid X_{1} \cup Y \cup S\right)\right] .
\end{aligned}
$$

For fixed $S \subset M$, a set of equations of the form of (17) results, the energy assigned to $X$ being $W(X \mid S)$. Therefore, by Lemma 3, the solution to Eq. (22) is

$$
\mu_{\Lambda \cup M}(X \cup S)=\mu_{M}(S) \exp [-W(X \mid S)] / Z_{n}(S) .
$$

Since the above holds for any natural $n$, we have found the DLR Eq. (9). Q.E.D.

Step 3: We conclude the proof of Theorem 1 with the case of infinite range interactions.

Let $\Lambda=[1, n]$, and fix a configuration $S \subset[n+1, \infty)$. Take $M=[n+1, n+m]$, $K=[n+m+1, n+m+k]$, and let $S_{M}=S \cap M$. Let $X \subset \Lambda, X \neq \emptyset$. Then the KS equations become [cf. Eq. (13)]

$$
\begin{aligned}
& \sum_{Y \subset A-X} \mu_{\Lambda \cup M}\left(X \cup Y \cup S_{M}\right) \\
& \quad=\lim _{k \rightarrow \infty} \sum_{\substack{Y \subset A-X \\
R \subset K}} \mu_{\Lambda \cup M \cup K}\left(X_{1} \cup Y \cup S_{M} \cup R\right) \exp \left[-W\left(x \mid X_{1} \cup Y \cup S_{M} \cup R\right)\right],
\end{aligned}
$$


where a recombination like in Eqs. (21) and (22) has been carried out. Now note, that, by Eq. (3),

$$
\left|\exp \left[-W\left(x \mid X_{1} \cup Y \cup S_{M} \cup R\right)\right]-\exp \left[-W\left(x \mid X_{1} \cup Y \cup S\right)\right]\right|<\varepsilon_{M},
$$

with $\varepsilon_{M}$ independent of $X, Y, S$ and $R$, and

Therefore

$$
\lim _{m \rightarrow \infty} \varepsilon_{M}=0 \text {. }
$$

$$
\begin{gathered}
\left|\sum_{Y \subset A-X} \mu_{\Lambda \cup M}\left(X \cup Y \cup S_{M}\right)-\sum_{Y \subset A-X} \mu_{\Lambda \cup M}\left(X_{1} \cup Y \cup S_{M}\right) \exp \left[-W\left(x \mid X_{1} \cup Y \cup S\right)\right]\right| \\
\leqq \varepsilon_{M} \mu_{M}\left(S_{M}\right) .
\end{gathered}
$$

Comparing Eqs. (24) and (17) and applying Lemma 3 (which in fact states that the $2^{n} \times 2^{n} \mathrm{KS}$ matrix is invertible), we find that

$$
\left|\mu_{A \cup M}\left(X \cup S_{M}\right)-\mu_{M}\left(S_{M}\right) \exp [-W(X \mid S)] / Z_{n}(S)\right| \leqq \delta_{M} \mu_{M}\left(S_{M}\right),
$$

with $\lim _{m \rightarrow \infty} \delta_{M}=0$. Since in the above $n$ is arbitrary, we have recovered the DLR Eq. (7).

Q.E.D.

\section{Complex Fugacity: The Spectrum of the KS Operator}

We now turn to the case of complex fugacity $z$. The KS equations and the DLR equations will be examined in the space $B$ of uniformly bounded correlation functions, Eq. (15).

Theorem. 4. (ia) For finite $L=[1, n], z^{-1}$ is in the spectrum of the KS matrix iff $z$ is a zero of one of the partition functions $Z_{n}$ or $Z_{k-1}(k \cup S), 2 \leqq k \leqq n, S \subset[k+1, n]$.

(ib) If $Z_{n}=0$, the $\mathrm{KS}$ equations have no solution, but the homogeneous equations have the solution

$$
\mu_{L}(X)=\exp [-U(X)] .
$$

(ic) If $Z_{k-1}(k \cup S)=0$ and $Z_{n} \neq 0$, the $\mathrm{KS}$ equations have the usual Gibbs solution. Moreover the homogeneous equations have a non-trivial solution, which is in the case of $S=[k+1, n]$ given by

$$
\begin{aligned}
& \mu_{L}(X \cup Y)=0, \quad X \subset[1, k-1], \quad Y \subset[k, n], \quad Y \neq[k, n] ; \\
& \mu_{L}(X \cup[k, n])=\exp [-W(X \mid[k, n])], \quad X \subset[1, k-1] .
\end{aligned}
$$

(For other $S$ the eigenvectors have a more complicated form.)

(ii) For infinite L, let $z$ be a zero of one of the partition functions

$$
Z_{k-1}(k \cup S \cup[p+1, \infty)), \quad 2 \leqq k \leqq p, \quad S \subset[k+1, p] .
$$

Then $z^{-1}$ is in the point spectrum of the $\mathrm{KS}$ operator. The corresponding eigenvector is given by

$$
\begin{aligned}
& \mu_{[1, N]}(X \cup S)=0, \quad N \geqq p, \quad X \subset[1, p], \quad S \subset[p+1, N], \quad S \neq[p+1, N] ; \\
& \mu_{[1, N]}(X \cup[p+1, N])=\gamma(X), \quad N \geqq p, \quad X \subset[1, p],
\end{aligned}
$$


where $\gamma(X)$ is the eigenvector of the $\mathrm{KS}$ operator for the finite volume $[1, p]$, with energy $W(X \mid[p+1, \infty))$ assigned to the configuration $X \subset[1, p]$.

(iii) Let the interactions be of finite range. Then the $\mathrm{KS}$ equations are equivalent to the DLR equations if none of the partition functions (26) is equal to zero.

Proof. (i) Let $\Delta_{n}$ be the $2^{n} \times 2^{n}$ determinant of the Eqs. (16) and (17). With the rearrangement of Eq. (17) into Eqs. (18)-(20) and the induction argument in the proof of Lemma 3, one readily sees that

$$
\Delta_{n}=\Delta_{n-1} \Delta_{n-1}(n) Z_{n} / Z_{n-1}
$$

$\Delta_{n-1}(n)$ differs from $\Delta_{n-1}$ in that the energy $U(X)$ is replaced by $W(X \mid n)$. Since $\Delta_{1}=Z_{1}$,

$$
\Delta_{n}=Z_{n} \prod_{k=2}^{n} \prod_{S \subset[k+1, n]} Z_{k-1}(k \cup S) .
$$

This proves (ia). The expressions for the eigenvectors in (ib) and (ic) can easily be verified.

(ii) With the KS equations expressed in the form (22) and (23) and with part (i) as a guide, one checks that Eq. (27) indeed solves the homogeneous KS equations, provided that the corresponding partition function is zero.

(iii) If $Z_{n}(S) \neq 0$, Step 2 in the proof of Theorem 2 remains valid here, by Theorem 4(ia). Even if $Z_{n}(S)=0$, Eq. (22) implies that

$$
\mu_{\Lambda \cup M}(X \cup S)=\mu_{\Lambda \cup M}(Y \cup S) \exp [-W(X \mid S)+W(Y \mid S)],
$$

by Theorem 4(ib). Equation (28) is the form, in which the DLR equations can reasonably be extended to complex interactions.

In Theorem 4(iii) we restrict ourselves to finite range interactions, because Step 3 in the proof of Theorem 2 does not extend as straightforwardly as Step 2. The subtle estimation (24) depends on the fact that we are dealing with states in that case.

It can be shown (cf. [8]), that in the space $B$ the EKS equations are equivalent to the DLR equations for all complex values of $z$. Theorem 4(iii) thus shows the effect of taking a smaller family of equations.

The spectrum of the KS operator for finite volumes has been studied by Pastur [9], for lattice systems as well as for continuous systems. He considered Eq. (14) not on the space $B$, but on the subspace of vectors $\varrho$ such that $K \varrho$ is independent of the particular order chosen on $L$. Solutions in this subspace in fact satisfy the EKS equations, and Pastur's result, that $z^{-1}$ is in the spectrum of $\boldsymbol{K}$ if $z$ is a zero of the partition function $Z_{n}$, is consistent with Theorem 4(ia).

It has also been shown by W. Klein [10], who studied continuous systems, that the KS operator for finite volumes has a pure point spectrum.

\section{Examples}

We first give an example of a situation, where the equilibrium state is unique, but where the DLR equations have more solutions in the space $B$, Eq. $(15)^{1}$.

${ }^{1}$ For one-dimensional systems with finite range interactions the DLR equations are known to have other solutions than states $[12,16]$; however, those solutions are never in $\mathrm{B}$ (the related remark in the conclusion of [12] is wrong). 
Example 1. Consider the Bethe lattice [11] (Fig. 1) with attractive nearest neighbor interaction:

$$
\begin{aligned}
& \Phi(x, y)=-J<0, \quad \text { if } x, y \text { are nearest neighbors } ; \\
& \Phi(X)=0, \quad \text { otherwise. }
\end{aligned}
$$

The interaction is invariant under the "translation" group, generated by three elements, $a, b, c$, with

$$
a^{2}=b^{2}=c^{2}=1 ; \quad a b \neq b a, \quad a c \neq c a, \quad b c \neq c b .
$$

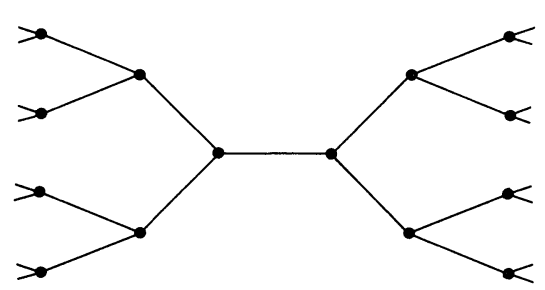

Fig. 1. The Bethe Lattice

A particularly of the Bethe lattice is, that for any two points $x, y$ there is a unique chain of nearest neighbors connecting $x$ and $y$. For an arbitrary set $X$ we define its connected completion $\bar{X}$ to consist, with each pair $x, y \in X$, of the whole chain connecting $x$ and $y$. A set $X$ is called connected if $\bar{X}=X$.

Let $\Lambda$ be a connected set. For $x \in \Lambda$, let $m(x)$ be the number of nearest neighbors of $x$ that lie outside $\Lambda$. The interior of $\Lambda$ is the collection of points $x \in \Lambda$ with $m(x)=0$; the border of $\Lambda$ consists of the points with $m(x)=1,2$, or $3(m(x)=3$ can only occur if $|\Lambda|=1$ ).

Now consider the expression

$$
\mu_{\Lambda}(X)=\mu_{\Lambda}(\emptyset) \exp [-U(X)] \prod_{x \in X}(q t)^{m(x)},
$$

where $q$ is a solution of the equation

$$
\left(z t^{4} q^{2}+1\right) /\left(z t^{2} q^{2}+1\right)=q t
$$

and where $t=\exp \left(\frac{1}{2} \beta J\right)$. Equation (30) is the equation occurring in the Bethe approximation for the case of three nearest neighbors. It guarantees that the $\mu_{\Lambda}(X)$, Eq. (29), satisfy the consistency relations (5). Thus, if $q \geqq 0$, Eq. (29) determines a state. Obviously, it is an equilibrium state, since $m(x) \neq 0$ only on the border of $\Lambda$.

Equations (29) and (30) immediately lead to the following property, familiar for one-dimensional systems [12]. Let $\Lambda_{1}, \Lambda_{2}$ be finite connected sets, such that $\Lambda_{1} \cap \Lambda_{2} \equiv \Lambda_{3} \neq \emptyset$. Let $X_{1} \subset \Lambda_{1}-\Lambda_{3}, X_{2} \subset \Lambda_{2}-\Lambda_{3}, X_{3} \subset \Lambda_{3}$. Then

$$
\mu_{\Lambda_{1} \cup \Lambda_{2}}\left(X_{1} \cup X_{2} \cup X_{3}\right)=\mu_{\Lambda_{1}}\left(X_{1} \cup X_{3}\right) \mu_{\Lambda_{2}}\left(X_{2} \cup X_{3}\right) / \mu_{\Lambda_{3}}\left(X_{3}\right) \text {. }
$$

Equation (31) remains true for arbitrary $\Lambda_{1}, \Lambda_{2}$ such that $\Lambda_{1} \cap \Lambda_{2}=\overline{\Lambda_{1}} \cap \overline{\Lambda_{2}}$; then both members of Eq. (31) for $\bar{\Lambda}_{1}, \bar{\Lambda}_{2}$ can be summed over all configurations in $\overline{\Lambda_{1}}-\Lambda_{1}$ and in $\overline{\Lambda_{2}}-\Lambda_{2}$ [with Eq. (5)]. 
Let us now restrict ourselves to $z=t^{-3}\left(\mu=-\frac{3}{2} J\right)$, the case of particle-hole symmetry. Then Eq. (30) becomes

$$
\left(t q^{2}+1\right) /\left(t+q^{2}\right)=q, \quad \text { or } \quad(q-1)\left[q^{2}-(t-1) q+1\right]=0 .
$$

If $t>3(\beta J>2 \log 3)$, there are three positive solutions to this equation, and, accordingly, three translation invariant equilibrium states (actually, there are many more non-invariant equilibrium states).

Lemma 5. If $t \leqq 3(\beta J \leqq 2 \log 3)$, Eq. (29) with $q=1$ gives the unique equilibrium state.

Proof. Consider the sequence of volumes $\left\{\Lambda_{n}\right\}$ as in Fig. 2. Let $B_{n}=\Lambda_{n}-\Lambda_{n-1}$; thus, $B_{n}$ is the border of $A_{n}$, and $m(x)=2$ for $x \in B_{n}$.

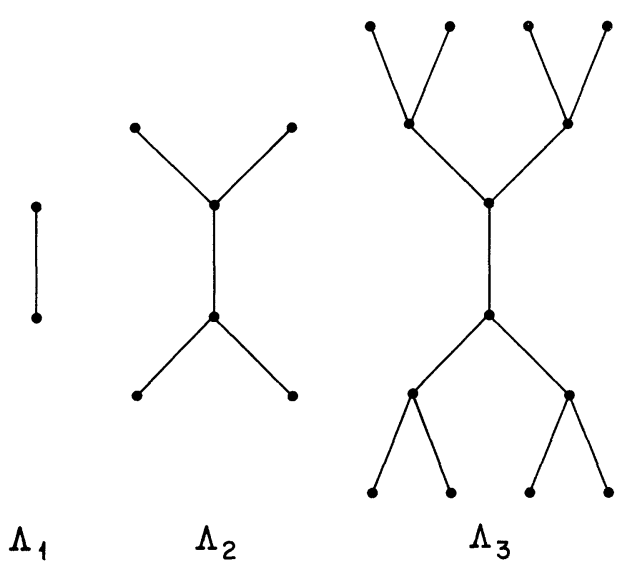

Fig. 2. The sequence of volumes $\left\{\Lambda_{n}\right\}$

Now consider the Gibbs state in the volume $\Lambda_{N}$, and let $\mu_{\Lambda_{n}}^{N}(X), X \subset \Lambda_{n}$, be the corresponding generalized correlation functions. As a boundary condition for $\Lambda_{N}$, fill $B_{N+1}$ either completely with particles or completely with holes. Then

where

$$
\mu_{\Lambda_{n}}^{N}(X)=\mu_{\Lambda_{n}}^{N}(\emptyset) \exp [-U(X)]\left(t q_{N-n}\right)^{2\left|X \cap B_{n}\right|},
$$

and where

$$
q_{j+1}=\left(t q_{j}^{2}+1\right) /\left(q_{j}^{2}+t\right),
$$

$$
\begin{array}{ll}
q_{0}=\mathrm{t}, & \text { for particle boundary condition } ; \\
q_{0}=\mathrm{t}^{-1}, & \text { for hole boundary condition. }
\end{array}
$$

Since for $t \leqq 3$ Eq. (27) has one real solution, in both cases

$$
\lim _{N \rightarrow \infty} q_{N-n}=1,
$$

so that for both boundary conditions the limiting state is given by Eq. (29) with $q=1$. 
Now by the Fortuin-Kasteleyn-Ginibre inequalities, the correlation functions $\varrho(X)$ for any boundary condition are in between those for particle and for hole boundary conditions (cf. [13]). Thus, the equilibrium state is unique. Q.E.D.

However, a complex solution of Eq. (32) still gives a solution of the DLR equations (although not a state). We show that this solution is in the space $B$, Eq. (16).

Lemma 6. The correlation functions $\varrho(X)$, computed with Eq. (29) and a complex solution q of Eq. (32), still satisfy

for $2.93<t<3$.

$$
|\varrho(X)| \leqq\left|q^{3}+1\right|^{-1}<0.55
$$

Proof. For $t<3$, the two complex solutions of Eq. (32) are

so that

$$
q=\frac{1}{2}(t-1) \pm \frac{1}{2} i[(3-t)(t+1)]^{\frac{1}{2}},
$$

$$
|q|=1 ; \quad\left|q^{3}+1\right|=(t-2)(t+1)^{\frac{1}{2}} ; \quad\left|t+q^{2}\right|=(t-1)(t+1)^{\frac{1}{2}} .
$$

If $X$ is connected, Eq. (29) or, more easily, Eq. (31) gives

so

$$
\varrho(X)=t^{|X|-1} q^{|X|+2} /\left(q^{3}+1\right)\left(t+q^{2}\right)^{|X|-1},
$$

$$
|\varrho(X)| \leqq\left|q^{3}+1\right|^{-1} .
$$

Now let $X$ be an arbitrary finite set, consisting of several connected pieces $X_{i}$. To form its completion $\bar{X}$, a number of connected sets $Y_{j}$ (called links) have to be added. A typical link $Y$ is pictured in Fig. 3. Let $Y$ be connected to $n \geqq 2$ of the sets $X_{i}$ in the points $(\oplus$ ), called hinges. (The hinges are in $X$, not in $Y$. A point may be a hinge of two links.) Then $Y$ consists of $n-2$ points with 3 nearest neighbors $(\bigcirc)$, called knots, and $2 n-3$ chains of arbitrary many points (-- -), connecting knots and hinges.

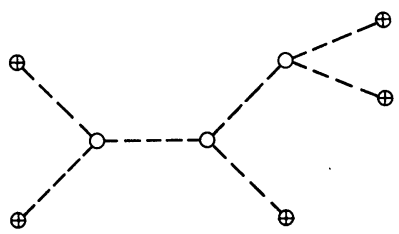

Fig. 3. A typical link with hinges $(\oplus)$, knots $(\bigcirc)$, and chains $(----)$

Let $H_{j}$ be the family of hinges of the link $Y_{j}$. We shall show that

$$
\left|\varrho\left(H_{j}\right)\right| \leqq\left|q^{3}+1\right|^{-1} .
$$

Then by repeated application of Eq. (31), for $\left|\Lambda_{3}\right|=1$, to the $X_{i}$ and $H_{j}, \varrho(X)$ for an arbitrary set can be estimated as

$$
|\varrho(X)| \leqq\left|q^{3}+1\right|^{-1},
$$

where use has been made of Eqs. (33) and (34) and of the fact that the density (one-point correlation function) satisfies

$$
\varrho(x)=q^{3}\left(q^{3}+1\right)^{-1} .
$$


Thus only Eq. (34) remains to be proved. Let $K$ be the collection of knots in the link $Y$, and let $H$ be the family of hinges of $Y$. Then

$$
\varrho(H)=\sum_{T \subset K} \mu_{H \cup K}(H \cup T) .
$$

Again using Eq. (31), each term on the right-hand side of Eq. (35) can be expressed in one- and two-point correlation functions.

The computation of the two-point correlation functions is a one-dimensional problem, solvable with the transfer matrix method. For two points $x, y$ with distance $d$,

This gives

$$
\begin{aligned}
& \varrho(x, y)=\left(q^{3 / 2} 0\right)\left(\begin{array}{cc}
q & t^{-1} q^{\frac{1}{2}} \\
t^{-1} q^{\frac{1}{2}} & 1
\end{array}\right)^{d}\left(\begin{array}{c}
q^{3 / 2} \\
0
\end{array}\right) Q^{-1}, \\
& Q=\left(q^{3 / 2} 1\right)\left(\begin{array}{cc}
q & t^{-1} q^{\frac{1}{2}} \\
t^{-1} q^{\frac{1}{2}} & 1
\end{array}\right)^{d}\left(\begin{array}{c}
q^{3 / 2} \\
1
\end{array}\right) \text {. }
\end{aligned}
$$

$$
\begin{aligned}
& \varrho(x, y)=q^{3}\left(q^{3}+1\right)^{-2}\left[q^{3}+(t-1)^{-d}\right]=\overline{\mu_{x, y}(\emptyset)} \\
& \mu_{x, y}(x)=q^{3}\left(q^{3}+1\right)^{-2}\left[1-(t-1)^{-d}\right] .
\end{aligned}
$$

Therefore we get the estimates

$$
\begin{aligned}
& |\varrho(x, y)|=\left|\mu_{x, y}(\emptyset)\right| \leqq t\left|t+q^{2}\right|^{-1}\left|q^{3}+1\right|^{-1} ; \\
& \left|\mu_{x, y}(x)\right| \leqq\left|q^{3}+1\right|^{-2} .
\end{aligned}
$$

Note that (36) is an equality if $x$ and $y$ are nearest neighbors $(d=1)$; (37) becomes an equality for $d \rightarrow \infty$.

The summation (35) can now be estimated by noting that instead of over $|H|-2$ knots one can sum over $|H|-2$ independent chains. Hence

$$
|\varrho(H)| \leqq\left|q^{3}+1\right|^{-1}\left(t\left|t+q^{2}\right|^{-1}\right)^{|H|-1}\left(t\left|t+q^{2}\right|^{-1}+\left|q^{3}+1\right|^{-1}\right)^{|H|-2} \leqq\left|q^{3}+1\right|^{-1} .
$$

It is the last crucial estimation that gives the lower bound 2.93 for $t$.

We conclude with two examples showing that there is no rigid connection between non-uniqueness of the equilibrium state and zeros of the partition function approaching the positive real axis.

Example 2. Consider the two-dimensional Ising model with attractive nearest neighbor interaction. Then at the critical temperature, the zeros of the partition function approach the positive real axis; however, the equilibrium state is unique there.

Example 3. Consider the two-dimensional Ising model with repulsive nearest neighbor interaction. Then for low temperatures there are fugacities $z_{1}, z_{2}$, such that there are two equilibrium states for $z_{1}<z<z_{2}$ [14], and such that the partition function is free of zeros for $z_{1}<|z|<z_{2}$ [15]. A lthough this last property has only been proved for special boundary conditions, we certainly expect it to remain true for the boundary conditions in Eq. (26), at least in the neighborhood of the positive real axis. 


\section{References}

1. Ruelle, D.: Statistical mechanics. New York: Benjamin 1969

2. Brascamp, H. J.: Commun. math. Phys. 18, 82-96 (1970)

3. Dobrushin, R. L.: Funct. Anal. Appl. 2, 292-301 (1968)

4. Lanford, O. E., Ruelle, D.: Commun. Math. Phys. 13, 194-215 (1969)

5. Gallavotti, G., Miracle-Sole, S.: Commun. Math. Phys. 7, 274-288 (1968)

6. Ruelle, D.: Commun. Math. Phys. 18, 127-159 (1970)

7. Gruber, C., Kunz, H.: Commun. Math. Phys. 22, 133-161 (1971)

8. Gruber, C., Lebowitz, J. L.: (Preprint) (E.P.F. L., Lausanne 1974)

9. Pastur, L.A.: (Preprint) (Institute for Theoretical Physics, A. N. Ukr. S.S.R., Kiev 1973)

10. Klein, W.: (Preprint) (M.I.T., Cambridge 1973)

11. Domb, C.: Advan. Phys. 9, 149-361 (1960), p. 284

12. Brascamp, H. J.: Commun. Math. Phys. 21, 56-70 (1971)

13. Ruelle, D.: Ann. Phys. 69, 364-374 (1972)

14. Dobrushin, R.L.: Funct. Anal. Appl. 2, 302-312 (1968)

15. Brascamp, H.J., Kunz, H.: Commun. math. Phys. 32, 93-106 (1973)

16. Gallavotti, G., Lebowitz, J. L.: Physica 70, 219-221 (1973)

Communicated by G. Gallavotti

H. J. Brascamp

Deptartment of Physics

Princeton University

Princeton, N.J. 08540, USA 
